University of Nebraska - Lincoln

DigitalCommons@University of Nebraska - Lincoln

8-17-2018

\title{
Histopathological, morphological, and molecular characterization of Sarcocystis species in elk (Cervus elaphus) from Pennsylvania, USA
}

\author{
Camila K. Cerqueira-Cézar \\ Beltsville Agricultural Research Center, Animal Parasitic Diseases Laboratory, Beltsville \\ Peter C. Thompson \\ Beltsville Agricultural Research Center, Animal Parasitic Diseases Laboratory, Beltsville \\ Fernando H.A. Murata \\ Beltsville Agricultural Research Center, Animal Parasitic Diseases Laboratory, Beltsville \\ Joseph Mowery \\ Beltsville Agricultural Research Center, Electron and Confocal Microscopy Unit, Building 12, Beltsville \\ Justin D. Brown \\ Pennsylvania Game Commission
}

See next page for additional authors

Follow this and additional works at: https://digitalcommons.unl.edu/usdaarsfacpub

Cerqueira-Cézar, Camila K.; Thompson, Peter C.; Murata, Fernando H.A.; Mowery, Joseph; Brown, Justin D.; Bafield, Jeremy; Rosenthal, Benjamin M.; and Dubey, J. P., "Histopathological, morphological, and molecular characterization of Sarcocystis species in elk (Cervus elaphus) from Pennsylvania, USA" (2018). Publications from USDA-ARS / UNL Faculty. 2229.

https://digitalcommons.unl.edu/usdaarsfacpub/2229

This Article is brought to you for free and open access by the U.S. Department of Agriculture: Agricultural Research Service, Lincoln, Nebraska at DigitalCommons@University of Nebraska - Lincoln. It has been accepted for inclusion in Publications from USDA-ARS / UNL Faculty by an authorized administrator of DigitalCommons@University of Nebraska - Lincoln. 
Authors

Camila K. Cerqueira-Cézar, Peter C. Thompson, Fernando H.A. Murata, Joseph Mowery, Justin D. Brown, Jeremy Bafield, Benjamin M. Rosenthal, and J. P. Dubey 


\title{
Histopathological, morphological, and molecular characterization of Sarcocystis species in elk (Cervus elaphus) from Pennsylvania, USA
}

\author{
Camila K. Cerqueira-Cézar ${ }^{1}$ • Peter C. Thompson ${ }^{1}$ • Fernando H. A. Murata ${ }^{1} \cdot$ Joseph Mowery $^{2}$ • Justin D. Brown ${ }^{3}$. \\ Jeremy Banfield ${ }^{3}$ • Benjamin M. Rosenthal ${ }^{1}$. Jitender P. Dubey ${ }^{1}$
}

Received: 30 April 2018 / Accepted: 2 July 2018 / Published online: 17 August 2018

(C) Springer-Verlag GmbH Germany, part of Springer Nature 2018

\begin{abstract}
Sarcocystis sarcocysts are common in many species of domestic and wild animals. Here, we report sarcocystosis in muscles from 91 free range elk (Cervus elaphus) from Pennsylvania, USA, tested by histopathology, transmission electron microscopy (TEM), and DNA sequencing. Sarcocysts were detected in hematoxylin and eosin (HE)-stained sections from 83 of 91 (91.2\%) elk, including 83/91 (91.2\%) tongues and 15/17 (88.2\%) hearts. With respect to age, sarcocysts were found in 0/5 calves, $8 / 9(88.8 \%)$ yearlings, and 75/77 (97.4\%) adults. Sarcocysts were identified in 62/69 (89.4\%) females and 21/22 (91.2\%) males. Associated lesions were mild and consisted of inflammatory foci around degenerate sarcocysts. There were two morphologically distinct sarcocysts based on wall thickness, thin $(<0.5 \mu \mathrm{m})$ and thick-walled $(>4.0 \mu \mathrm{m})$. Thin-walled sarcocysts had a TEM "type 2" and villar protrusions (vps), identical to Sarcocystis wapiti previously described from elk in western USA. This species was present both in tongue and heart samples and was detected in all infected elk. Thick-walled sarcocysts consisted of three morphologic variants, referred to herein as subkinds A, B, C. Subkind A sarcocysts were rare; only four sarcocysts were found in three elk. Histologically, they had a 5-8- $\mu$ m thick wall with tufted vp. By TEM, the sarcocyst wall was "type 12 " and appeared similar to Sarcocystis sybillensis, previously described from elk in USA. Subkind B, Sarcocystis sp.1 sarcocysts were also rare, found in

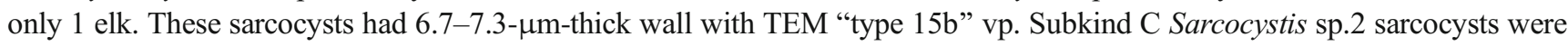
more common (22/91). Morphologically, the sarcocyst wall was 6.1-6.8 $\mu \mathrm{m}$ thick and contained "type 10b" vp. Comparisons of ribosomal DNA loci with published sequences indicated all sarcocysts were similar to what has previously been isolated from cervid hosts across the northern hemisphere. Phylogenetic analysis placed the thin-walled $S$. wapiti within a strongly supported clade with $S$. linearis and $S$. taeniata, while the thick-walled cysts were very closely related to S. truncata, S. elongata, S. silva, and $S$. tarandi. Further sequencing is needed to produce molecular diagnostics to distinguish among these species. North American elk are hosts to multiple Sarcocystis species with diverse morphology, deriving from two separate evolutionary lineages.
\end{abstract}

Keywords Elk (Cervus elaphus) $\cdot$ Sarcocystis $\cdot$ Ultrastructure $\cdot$ Phylogeny

Section Editor: Daniel K Howe

Jitender P. Dubey

jitender.dubey@ars.usda.gov

1 United States Department of Agriculture, Agricultural Research Service, Beltsville Agricultural Research Center, Animal Parasitic Diseases Laboratory, Beltsville, MD 20705-2350, USA

2 United States Department of Agriculture, Agricultural Research Service, Beltsville Agricultural Research Center, Electron and Confocal Microscopy Unit, Building 12, Beltsville, MD 20705-2350, USA

3 Pennsylvania Game Commission, 2001 Elmerton Avenue, Harrisburg, PA 17110-9797, USA

\section{Introduction}

Prior to European settlement, elk (Cervus elaphus, also called wapiti) inhabited nearly all of Pennsylvania, with the highest densities occurring in the Alleghany Mountains (Williams et al. 1985). Like many eastern states, rapid expansion and exploitation by early immigrants along with habitat changes caused the elk population to diminish across the state, and by most accounts, elk had been hunted to local extinction by 1877 (Williams et al. 1985). From 1913 to 1926,177 elk were reintroduced to nine Pennsylvania counties from multiple locations including Yellowstone National Park, Montana, private reserves in Pennsylvania, and Wind Cave National 
Park, South Dakota. The first state agency survey was conducted in 1971 at which time around 65 elk were present in Pennsylvania (Eveland et al. 1979). Since then, surveys have been conducted annually and the population has grown to a minimum count of 950 in 2016 . This growth is largely a result of habitat management in the $9731 \mathrm{~km}$ Elk Management Area that encompasses all of Cameron and parts of Elk, Clinton, Potter, Clearfield, Tioga, Jefferson, Lycoming and McKean counties.

Sarcocystis spp. are globally distributed protozoan parasites that infect a wide diversity of mammals, reptiles, and birds. Over 200 species of Sarcocystis have been described, which vary in their pathogenicity for the host, ranging from avirulent to severe disease; some species are zoonotic (Dubey et al. 2015, 2016). Among wild mammals, relatively little is known of Sarcocystis infection in the elk (Dubey et al. 2016). Sarcocysts have been sporadically reported from elk in North America (Mahrt and Colwell 1980; Sayama 1952; Pond and Speer 1979; Lagerquist and Foreyt 1993). Two species of Sarcocystis, S. wapiti (Speer and Dubey 1982), and $S$. sybillensis (Dubey et al. 1983) have been described from Western elk in North America (Dubey et al. 1983; Lagerquist and Foreyt 1993). Sarcocystis-associated clinical disease has not been reported from naturally infected elk, but experimentally infected elk had reduced weight gain with higher parasite burdens (Dubey et al. 1983; Foreyt et al. 1995).

Here, we report high prevalence of Sarcocystis spp. from elk from Pennsylvania and provide detailed morphological characterization and initial genetic characterization.

\section{Material and methods}

\section{Samples collection}

During 2016 Pennsylvania hunting season, 91 free-ranging elk tongue samples were collected from 77 adults, 9 yearlings, and 5 calves and tested for protozoal infections. Results of testing for Toxoplasma gondii infection were published earlier (Dubey et al. 2017). The same samples were used for testing for Sarcocystis infection.

Hearts were collected from 17 of these elk. Data on age, sex, and county were recorded (Table 1). Samples were collected at the mandatory check station within $24 \mathrm{~h}$ of harvest. Heart and tongue samples were maintained at $4{ }^{\circ} \mathrm{C}$ after collection and submitted within $48 \mathrm{~h}$ of harvest to Animal Parasitic Diseases Laboratory, USDA, Beltsville, Maryland for testing.

\section{Testing for Sarcocystis spp. infection}

Muscle samples from 91 tongue and 17 hearts were tested for Sarcocystis using direct microscopic examination, histologically, and by transmission electron microscopy (TEM). For direct microscopy, 1-3-mm pieces of tongue muscle were squashed between slides and coverslips and examined at $\times$ 100-400 magnification.

For histological examination, muscles were fixed in $10 \%$ buffered formalin, embedded in paraffin, sectioned at $5.0 \mu \mathrm{m}$, and stained with hematoxylin and eosin (HE). Slides were examined microscopically, and measurements were made digitally with Olympus DP73 camera.

For TEM, individual cysts from paraffin blocks were excised and processed as described previously (Trupkiewicz et al. 2016).

\section{Molecular characterization}

Molecular characterization was conducted on six sarcocysts (four thick-walled and two thin-walled) from six different elk. Each sample was placed individually in $20 \mu \mathrm{l}$ of saline $(0.85 \%)$ and subjected to DNA isolation using DNeasy Blood and Tissue Kit (Qiagen Inc., Valencia, CA, USA) according to manufacturer's instructions.

DNA characterization was conducted by PCR amplification and sequencing of $18 S, 28 S$ and ITS-1 ribosomal DNA. Each locus was amplified from thick-walled sarcocysts using
Table 1 Prevalence of Sarcocystis sarcocysts among elks in Pennsylvania, USA, by histopathology

\begin{tabular}{|c|c|c|c|c|c|c|c|}
\hline \multirow[t]{2}{*}{ Variables } & & \multicolumn{3}{|l|}{ Tongue } & \multicolumn{3}{|l|}{ Heart } \\
\hline & & Sampled & Positive & $\%$ positive & Sampled & Positive & $\%$ positive \\
\hline \multirow[t]{4}{*}{ Age } & Adult & 77 & 75 & 97.4 & 13 & 13 & 100.0 \\
\hline & Yearling & 9 & 8 & 88.8 & 2 & 2 & 100.0 \\
\hline & Calf & 5 & 0 & 0.0 & 2 & 0 & 0.0 \\
\hline & Total & 91 & 83 & 91.2 & 17 & 15 & 88.2 \\
\hline \multirow[t]{3}{*}{ Sex } & Female & 69 & 62 & 89.8 & 13 & 11 & 84.6 \\
\hline & Male & 22 & 21 & 95.4 & 4 & 4 & 100.0 \\
\hline & Total & 91 & 83 & 91.2 & 17 & 15 & 88.2 \\
\hline \multirow[t]{5}{*}{ County } & Cameron & 16 & 14 & 87.5 & 4 & 3 & 75.0 \\
\hline & Centre & 6 & 5 & 83.3 & 0 & 0 & 0 \\
\hline & Clearfield & 16 & 15 & 93.7 & 2 & 2 & 100.0 \\
\hline & Elk & 53 & 49 & 92.4 & 11 & 9 & 81.8 \\
\hline & Total & 91 & 83 & 91.2 & 17 & 15 & 88.2 \\
\hline
\end{tabular}



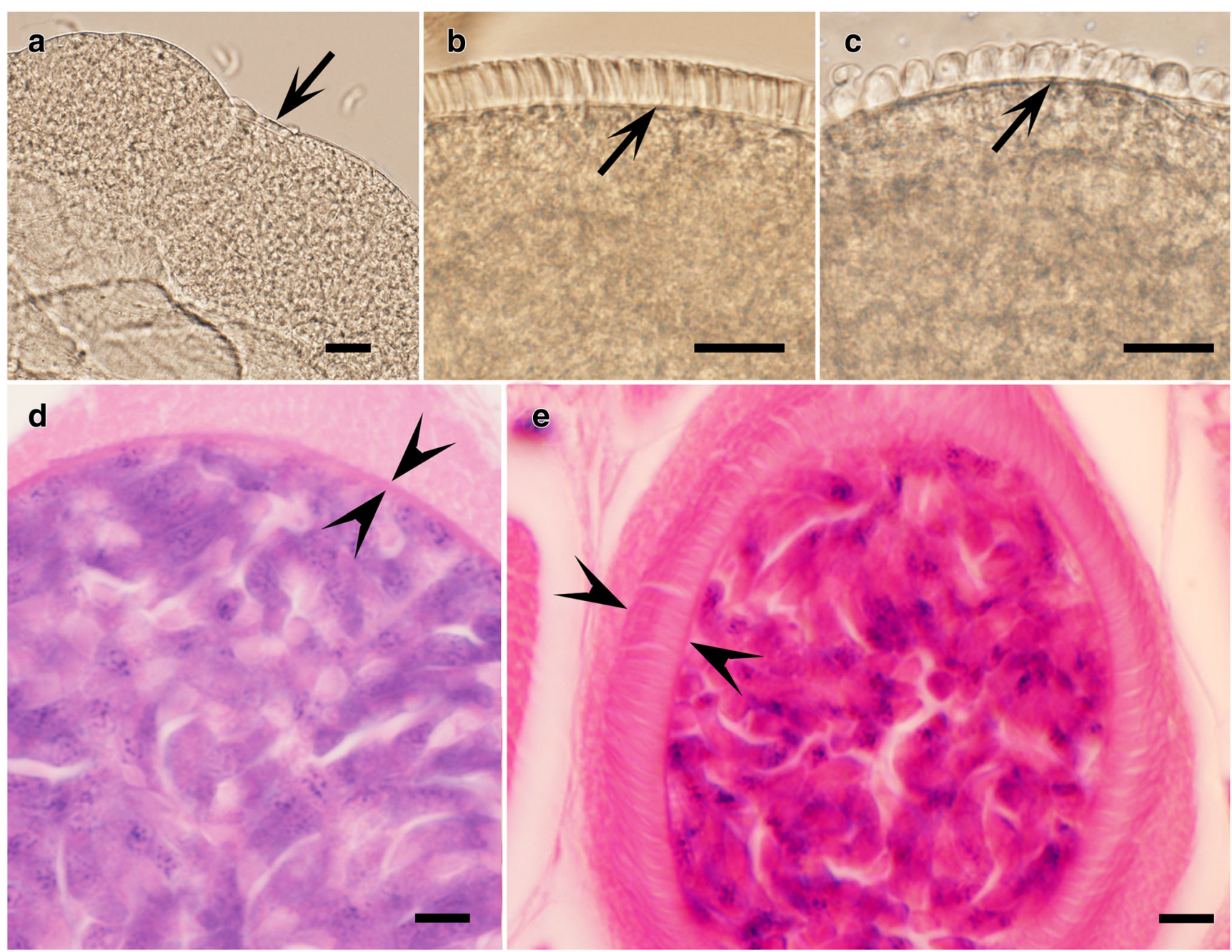

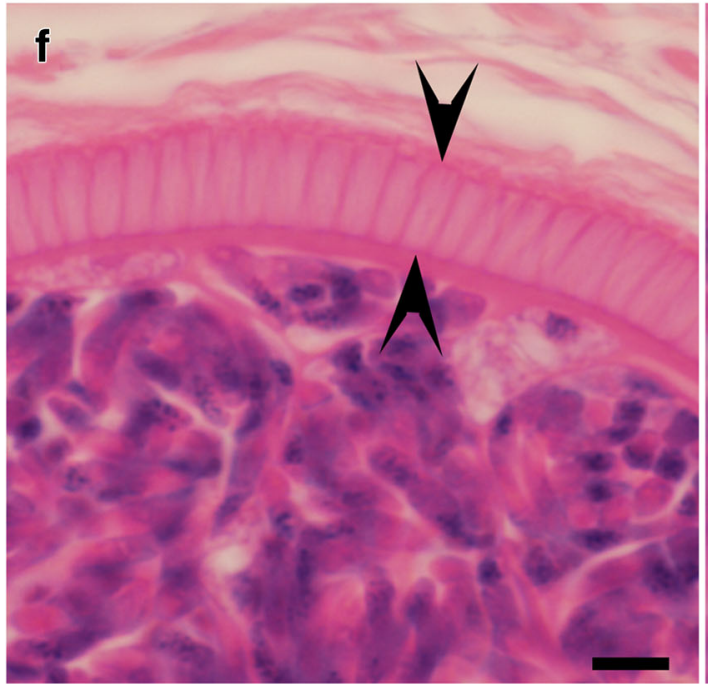

Fig. 1 Sarcocysts in tongues of naturally infected elk. a-c Squash preparations of cyst walls, unstained. $\mathbf{d}-\mathbf{g}$ Histological sections stained with hematoxylin and eosin (HE). Scale bars in a-c $20 \mu \mathrm{m}, \mathbf{d}-\mathbf{g} 5 \mu \mathrm{m}$. a Sarcocystis wapiti. Note thin sarcocyst wall, without any protrusions (arrow). b Subkind C Sarcocystis sp.2. Note upright villar protrusions (vps, arrow). c Sarcocystis sp.1, subkind B with molar teeth-like vp

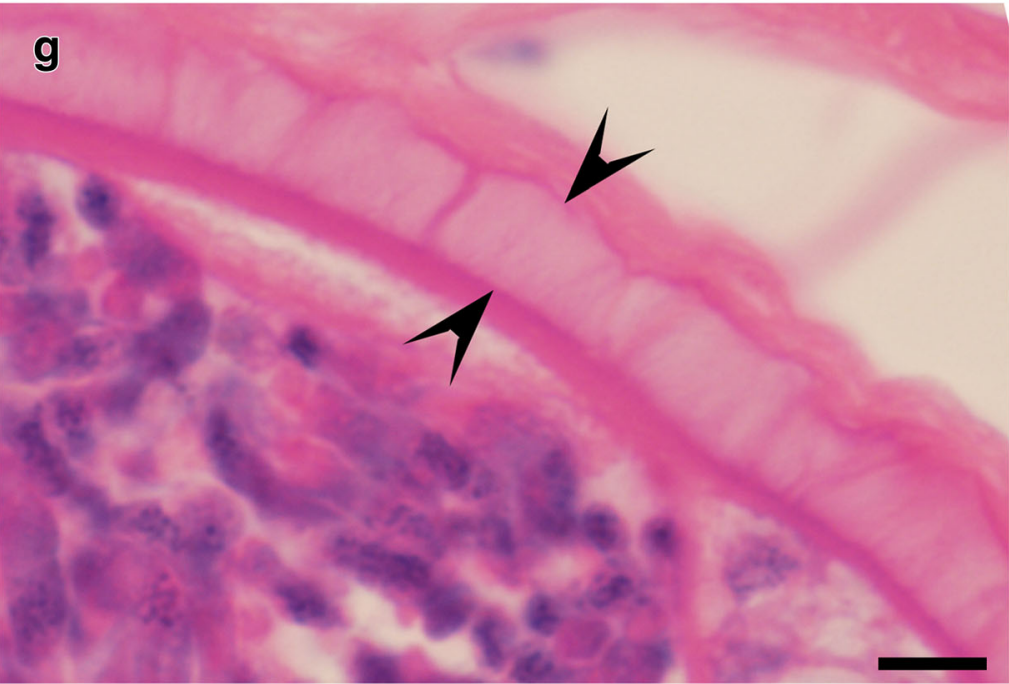

(arrow). d Sarcocystis wapiti. Note thin sarcocyst wall (opposing arrowheads). e Sarcocystis sybillensis-like. Note thick sarcocyst wall with tuftlike vp that stain darker at the tips (opposing arrowheads). f Note upright vp (opposing arrowheads). g Subkind B Sarcocystis sp. one with molar teeth-like vp (opposing arrowheads) 


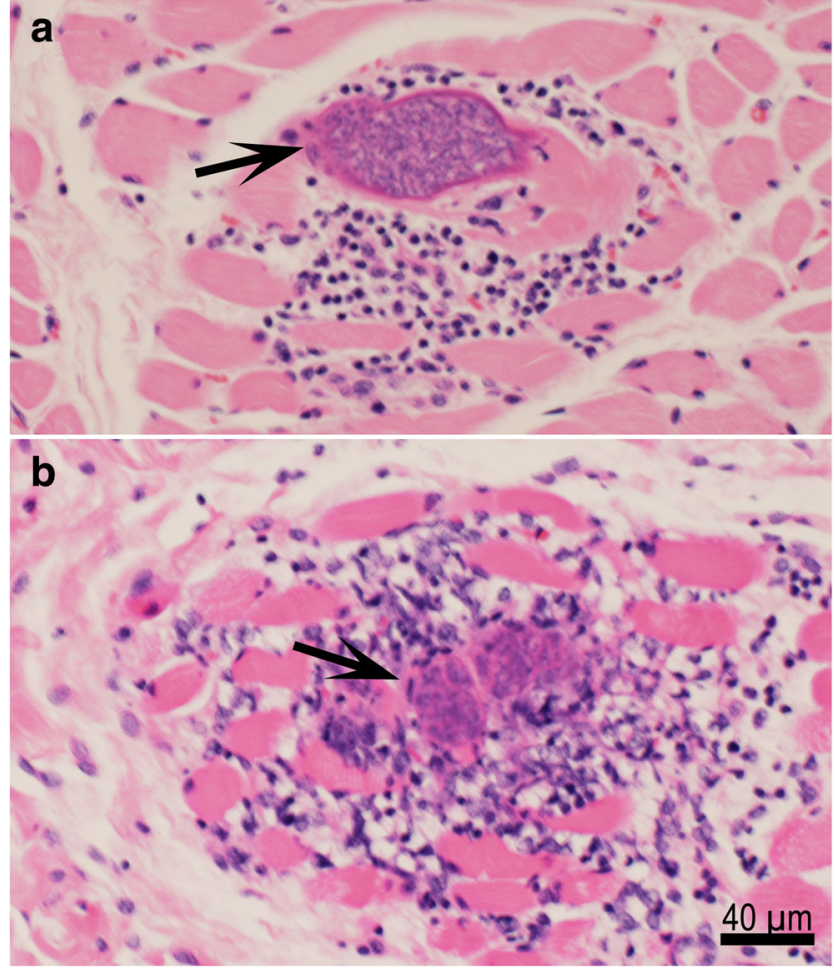

Fig. 2 Myositis in tongue of Sarcocystis-infected elk. Hematoxylin and eosin stain (HE). Bar scale applies to both parts. a Mononuclear cell infiltration associated with a degenerating thick-walled sarcocyst (arrow). b Degeneration of sarcocyst (arrow) and myocytes

previously described methods (Cerqueira-Cézar et al. 2017; Gjerde and Schulze 2014). However, amplification from thin-walled cysts was only possible for the $18 \mathrm{~S}$ locus. Amplified fragments were sequenced using amplification primers with BigDye Terminator v3.1 cycle sequencing (Applied Biosystems, Foster City, CA, USA). Sequencing products were analyzed on an ABI 3730 Sequencer.

Sequencing chromatograms were examined in Geneious R11.0.5 (Biomatters Limited, New Zealand), and consensus sequences for each sample were generated only from areas where both forward and reverse sequencing were present. Consensus sequences were then compared to other Sarcocystis sequences deposited in GenBank (https://www. ncbi.nlm.nih.gov/genbank).

In order to establish phylogenetic relationships between Sarcocystis species, $18 S$ sequences were aligned using the Geneious v.11.0.5 MAFFT; plug-in and neighbor-joining trees were built with the HKY substitution model in the Geneious v.11.0.5 Tree Builder. Consensus trees were constructed from alignments of 970 sites from $18 S$ rDNA for 20 taxa. After removing insertions, deletions, and indeterminate base calls, trees were built from 603 homologous sites. Support for phylogenetic trees was assessed using 1000 bootstrap replicates.
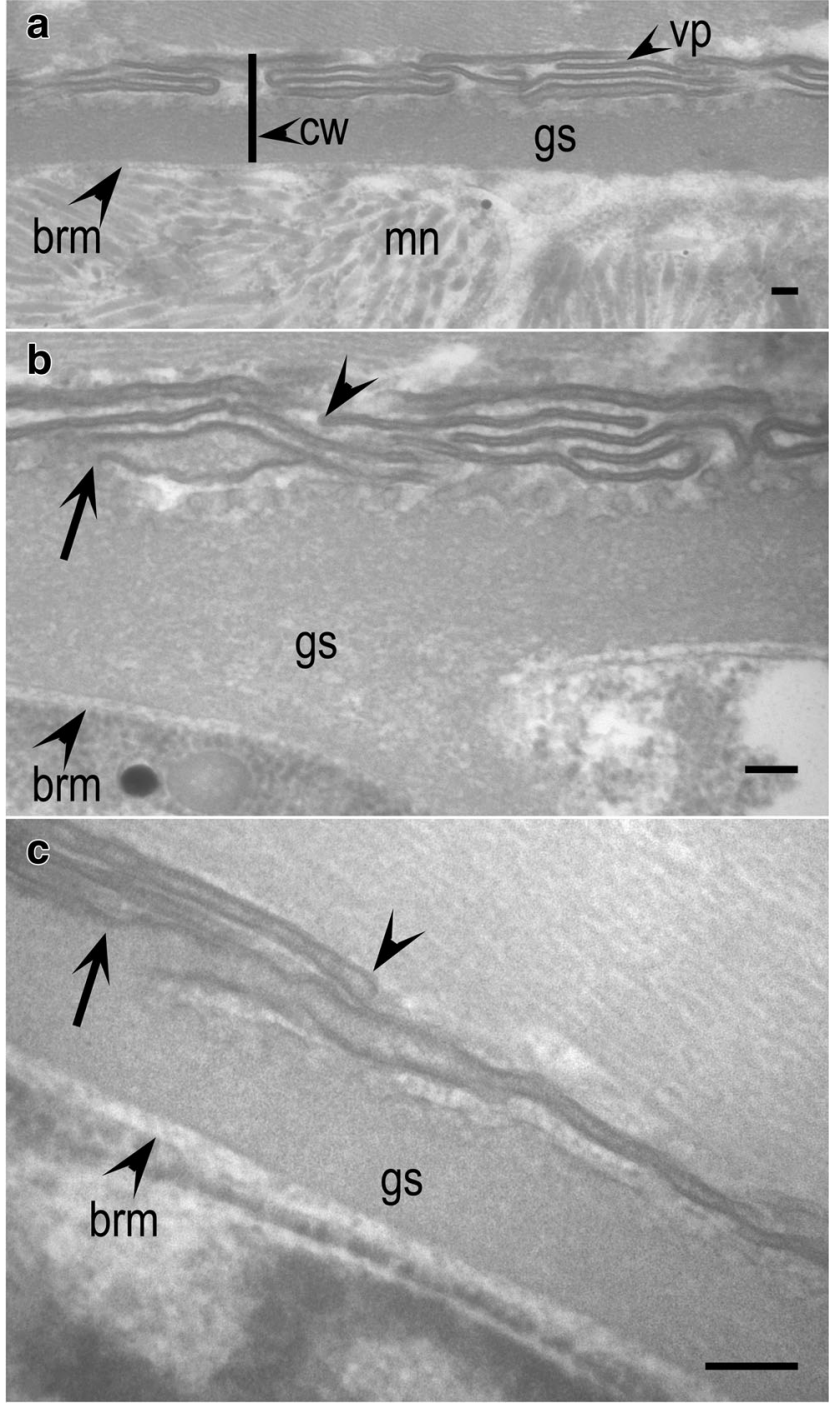

Fig. 3 TEM of Sarcocystis wapiti sarcocyst walls in three naturally infected elk. Scale bars in all three parts $=100 \mathrm{~nm}$. Note thin cyst wall (cw), villar protrusions (vps), ground substance layer (gs), bradyzoite pellicle membrane (brm), and micronemes $(\mathrm{mn})$ in bradyzoites. The total thickness of the wall, including the gs is $<0.5 \mu \mathrm{m}$. a Note vps are folded over the sarcocyst wall. b, c Note the vp are wider at the base (arrow), and narrow at the tip, which is blind (arrowhead)

\section{Results}

\section{Light microscopic observations}

Sarcocysts (Fig. 1) were detected in HE-stained sections from 83/ 91 (91.2\%) elk, including all tongue samples and 15/17 (88.2\%) hearts. With respect to age, sarcocysts were identified in $8 / 9$ (88.8\%) yearlings, $75 / 77(97.4 \%)$ adults, and 0/5 calves. Sarcocysts were detected in 62/69 (89.4\%) females and 21/22 (91.2\%) males (Table 1). Associated lesions were mild and consisted of inflammatory foci around degenerate sarcocysts (Fig. 2). Prevalence of Sarcocystis was similar in elk from four 


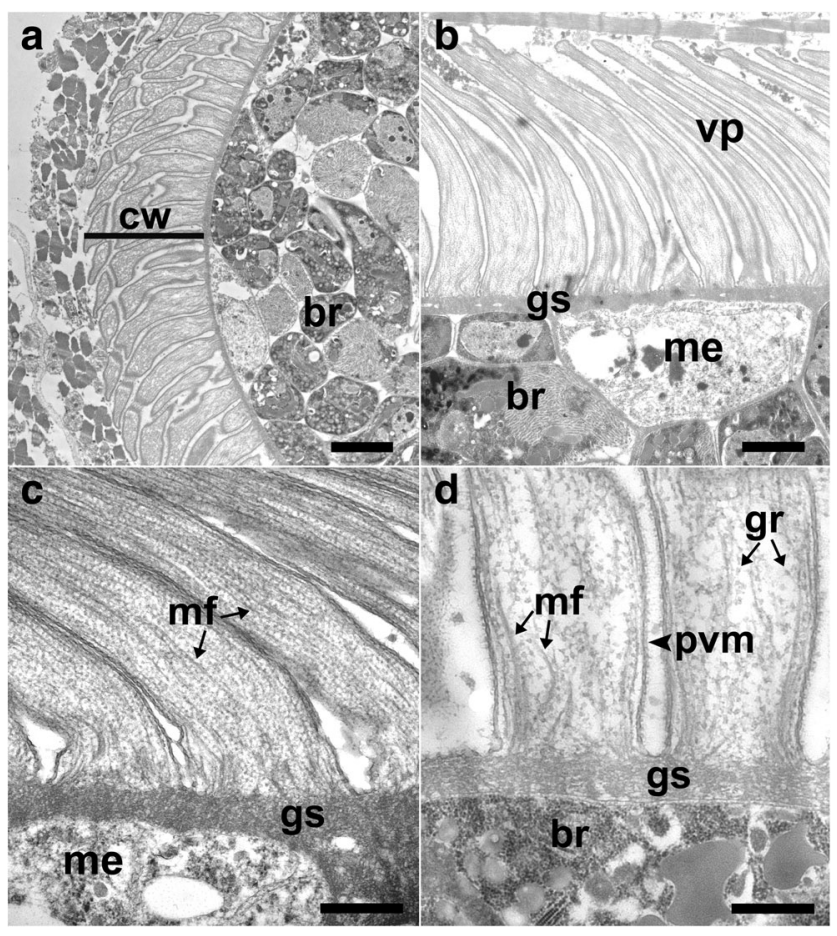

Fig. 4 TEM of subkind A Sarcocystis sybillensis-like sarcocyst. Scale bar in a $2.5 \mu \mathrm{m}, \mathbf{b} 2.0 \mu \mathrm{m}, \mathbf{c}, \mathbf{d} 500 \mathrm{~nm}$. Note bradyzoites (br), cyst wall (cw), granules (gr), ground substance layer (gs), metrocyte (me), microfilaments (mf), parasitophorous vacuolar membrane (pvm), villar protrusion (vp). a Note thick-walled cyst with curved and sometimes folded vp, and numerous br. b Note curved vp, which is thicker at the base and thinner at the top, with a blind end. Note thin gs and presence of me and br. c Note vp base with $\mathrm{mf}$, which is denser on the top of vp. Also, note gs and me. $\mathbf{d}$ High magnification of vp base, with details of $\mathrm{mf}$, gr, pvm, gs and br

counties of Pennsylvania (87\% of 16 from Cameron, $83.3 \%$ of 6 from Centre, $93.7 \%$ of 16 from Clearfield, and $92.4 \%$ of 53 from Elk).

Microscopically, there were 2 morphologically distinct sarcocysts and thin $(<0.5 \mu \mathrm{m})$ and thick-walled $(>4.0 \mu \mathrm{m})$. The thick-walled sarcocysts had three subkinds (A, B, C).

\section{Thin-walled Sarcocystis wapiti sarcocysts}

Thin-walled sarcocysts were found in all infected elk. In fresh unstained tissue squashes and HE-stained sections, sarcocysts had a smooth wall, without any villar protrusions (vps) (Fig. 1a, d). In tissue squashes, they were up to $1525 \mu \mathrm{m}$ long and up to $153 \mu \mathrm{m}$ wide. Sarcocysts in tongue were longer than in the heart. Only thin-walled sarcocysts were found in the heart. These sarcocysts were identified in the myocardium, pericardium, and in Purkinje fibers of the heart.

Seven thin-walled sarcocysts from three elk were examined by TEM. They had a TEM "type 2" (Dubey et al. 2016) wall with slender vp folded on the wall (Fig. 3). The length of the vp was difficult to measure because of folding. The vp ends were blunt without evidence of bifurcation (Fig. 3). These sarcocysts

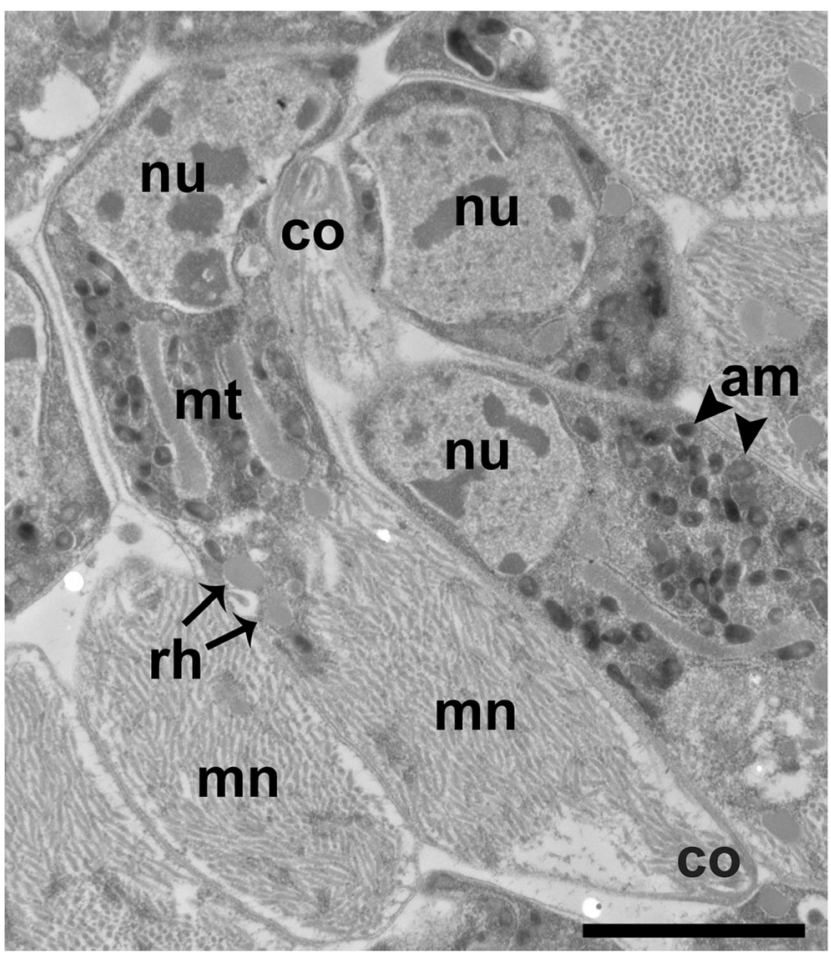

Fig. 5 TEM of subkind A Sarcocytis sybillensis-like bradyzoites. Note amylopectin granules (am), conoid (co), micronemes ( $\mathrm{mn}$ ), mitochondria $(\mathrm{mt})$, nucleus $(\mathrm{nu})$, and rhoptries $(\mathrm{rh})$

were identical to that described for $S$. wapiti by Speer and Dubey (1982).

\section{Thick-walled subkind A Sarcocystis sybillensis-like sarcocysts}

These sarcocysts were rarely detected; a total of four sarcocysts were observed in an adult male and an adult female from Cameron County and an adult female from Clearfield County. Histologically, three sarcocysts in cross sections were $57 \times 48,69 \times 50$, and $81 \times 50 \mu \mathrm{m}$, and the fourth longitudinally cut sarcocyst was $228 \times 60 \mu \mathrm{m}$. They had a 5-8- $\mu \mathrm{m}$-thick "type 12" wall, with tufted vp, the tips of vp stained darker with HE stain (Fig. 1e).

The three sarcocysts examined with TEM had a wall that was $6.3 \mu \mathrm{m}$ thick $(5.7-7.5 \mu \mathrm{m})$. The vps were $5.2-7.3 \mu \mathrm{m}$ long, curved, and sometimes folded (Fig. 4). The vps were thicker at the base $(681 \mathrm{~nm} ; 500-863 \mathrm{~nm})$ and tapered distally (331 nm; $242-421 \mathrm{~nm}$ ), without any visible branching. The microfilaments in vp varied in density, being denser in the middle of vp. Fine granules were present on filaments and appeared linear. The gs layer was smooth and lacked microtubules or granules, measuring between 0.4 and $1 \mu \mathrm{m}$. The bradyzoites were up to $9.5 \mu \mathrm{m}$ long and $2.3 \mu \mathrm{m}$ wide, with nucleus, conoid, mitochondria, micronemes, amylopectin granules, and rhoptries (Fig. 5). In general, subkind A sarcocysts were similar to $S$. sybillensis sarcocysts 


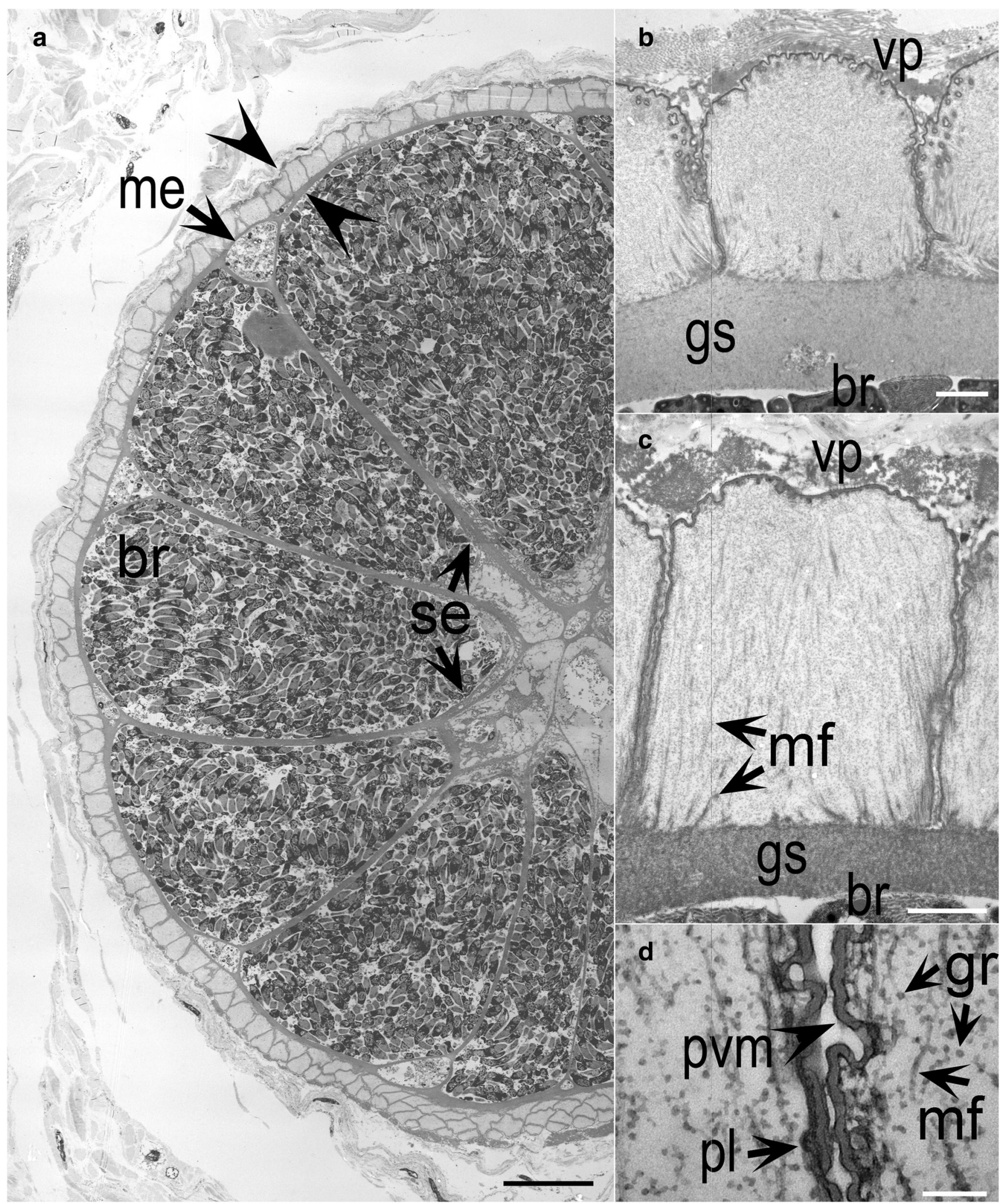

Fig. 6 TEM of subkind B Sarcocystis sp.1 sarcocysts. Scale bar in a $20 \mu \mathrm{m}, \mathbf{b}, \mathbf{c} 1 \mu \mathrm{m}, \mathbf{d} 100 \mathrm{~nm}$. Note bradyzoite (br), ground substance layer (gs), metrocyte (me), microfilament (mf), plaque (pl), parasitophorous vacuolar membrane (pvm), septa (se), villar protusion (vp). a Part of a sarcocyst with thick wall (opposing arrowheads). The sarcocyst is divided by thick se enclosing numerous br and a few me. The center of the sarcocyst is degenerating. b, c Thick sarcocyst wall with tombstone-like vp and thick gs. The pvm is wavy on all three unattached sides. The vp contain fine $\mathrm{mf}$, which are dense at the base. $\mathbf{d}$ Details of the pvm along the side of $2 \mathrm{vp}$. The pvm is invaginated and has electrondense pl. Also, note $\mathrm{mf}$ and fine gr 


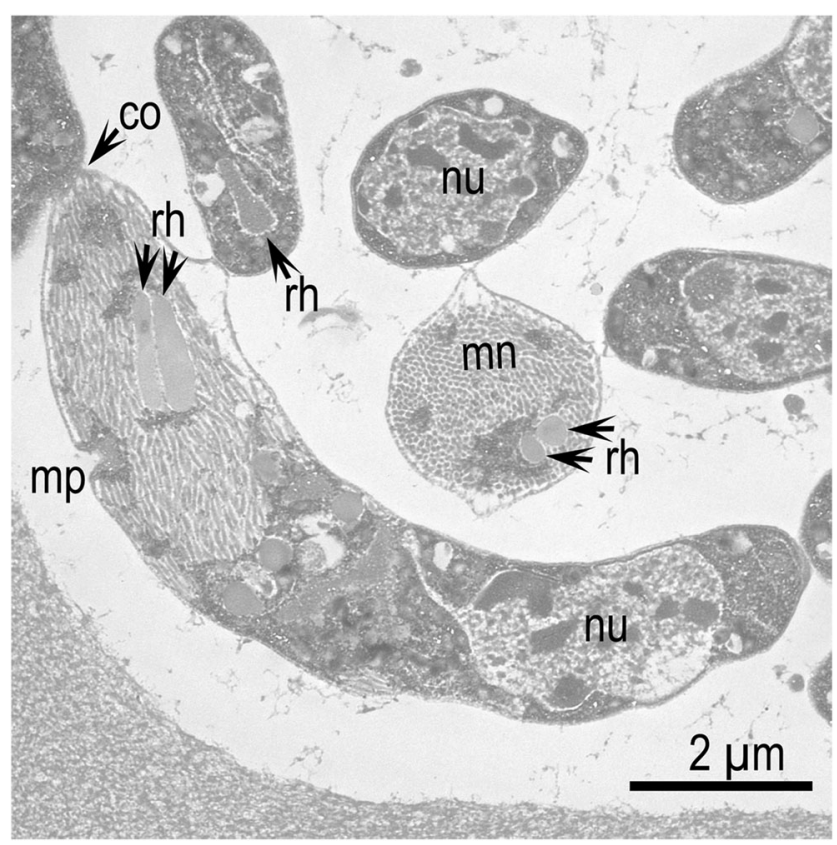

Fig. 7 TEM of subkind B Sarcocystis sp.1 bradyzoites. Note the conoid (co), two rhoptries (rh), numerous micronemes (nm), micropore (mp), and a subterminal nucleus (nu)

previously described from Western elk in North America (Dubey et al. 1983).

\section{Unidentified thick-walled subkind B Sarcocystis sp.1 sarcocysts}

Subkind B sarcocysts were rare; three (one in fresh muscle squash, two in HE-stained sections) were detected in one adult female elk from Elk County (Fig. 1c, g). The only subkind B sarcocyst detected in a muscle squash (Fig. 1c) was photographed and processed for DNA extraction. In HE-stained sections, these sarcocysts were up to $480 \times$ $326 \mu \mathrm{m}$ and had $4 \mu \mathrm{m}$ thick wall with molar teeth-like vp (Fig. 1g).

The two sarcocysts examined with TEM had a parasitophorous vacuolar membrane (pvm) that was wavy with invaginations along the three unattached (free) sides of the vp (Fig. 6). The vps were rectangular, tombstone-like type with TEM type "15b" wall (Dubey et al. 2016); they were 4.9-5.5 $\mu \mathrm{m}$ long and up to $4.6 \mu \mathrm{m}$ wide. Some vps were wider than long. The vp contained microfilaments that were denser at the base and had fine granules (Fig. $6 \mathrm{c}, \mathrm{d})$. The ground substance layer (gs) was uneven and up to $1.8 \mu \mathrm{m}$ thick. The total thickness of the sarcocyst wall including the vp and gs was up to $7.0 \mu \mathrm{m}$ thick, approximately. Bradyzoites were 7-10 $\mu \mathrm{m}$ long and 2.0 $2.8 \mu \mathrm{m}$ wide (Fig. 7) and contained nucleus, numerous micronemes, two rhoptries, a micropore, and a conoid. The micronemes were present in conoidal third of the bradyzoite.
Unidentified thick-walled subkind C Sarcocystis sp. 2 sarcocysts

Subkind C sarcocysts were found in 22 elk. In fresh unstained muscle squashes (Fig. 1b) and in HE-stained sections (Fig. 1f), the sarcocyst wall was 6.1-6.8 $\mu \mathrm{m}$ thick with upright elongated "type10b" vp. The sarcocysts were $315 \mu \mathrm{m}$ long $(120-950 \mu \mathrm{m})$ and $143 \mu \mathrm{m}$ wide $(45-280 \mu \mathrm{m})$.

Five sarcocysts examined with TEM had a pvm that was wavy with invaginations along the three unattached (free) sides of the vp and lined by a 15-20-nm-thick electron dense layer (edl) (Fig. 8). A few electrondense plaques (pls) were seen on pvm (Fig. 8d). The vps were elongated, upright, 5.0-6.2 $\mu \mathrm{m}$ long, and 1.2$2.0 \mu \mathrm{m}$ wide. They contained fine microfilaments that were denser at the base than the tips. A few granules were present in pv core. The gs layer was smooth, without microtubules and approximately $1 \mu \mathrm{m}$ thick. The total thickness of the sarcocyst wall including the $\mathrm{vp}$ and gs was $6.1-6.8 \mu \mathrm{m}$. Bradyzoites were $8.1 \mu \mathrm{m}$ long (7.2-10.8) and contained prominent mitochondria, nucleus, numerous micronemes, amylopectin granules, rhoptries, and a conoid (Fig. 9).

\section{Molecular analyses}

Partial DNA sequences were generated for $18 S \mathrm{rDNA}$, $28 S$ rDNA, and ITS-1 from thick-walled sarcocysts. Only a small region of $18 \mathrm{~S}$ was successfully sequenced from thin-walled cysts. Sequences from thick-walled cysts were submitted to NCBI GenBank and given the accession numbers MH167458 to MH167460 for the $18 S$ rDNA locus, MH157913 for ITS-1, and MH157244 to MH157246 for 28S. Partial 18S sequences from three thin-walled sarcocysts were given the accession numbers MH167455 to 167457.

\section{$18 \mathrm{~S}$ ribosomal DNA sequences}

Despite multiple attempts, most samples did not sequence well at the $18 \mathrm{~S} r D N A$ locus. Nevertheless, 603 homologous bases from $18 \mathrm{~S}$ were obtained from two thick-walled cysts and two thin-walled cysts. In neighbor-joining trees, all isolates were contained in a well-supported clade (100\% bootstrap support) with other cervid Sarcocystis species. Thickwalled cysts formed a group near the base of the clade with S. elongata, S. truncata, S. silva, and S. tarandi, while sequences from thin-walled cysts were within a strongly supported sub-clade (100\% bootstrap support) containing $S$. taeniata and S. linearis (Fig. 10). All sequences within either group had greater than $98 \%$ identical sites. 


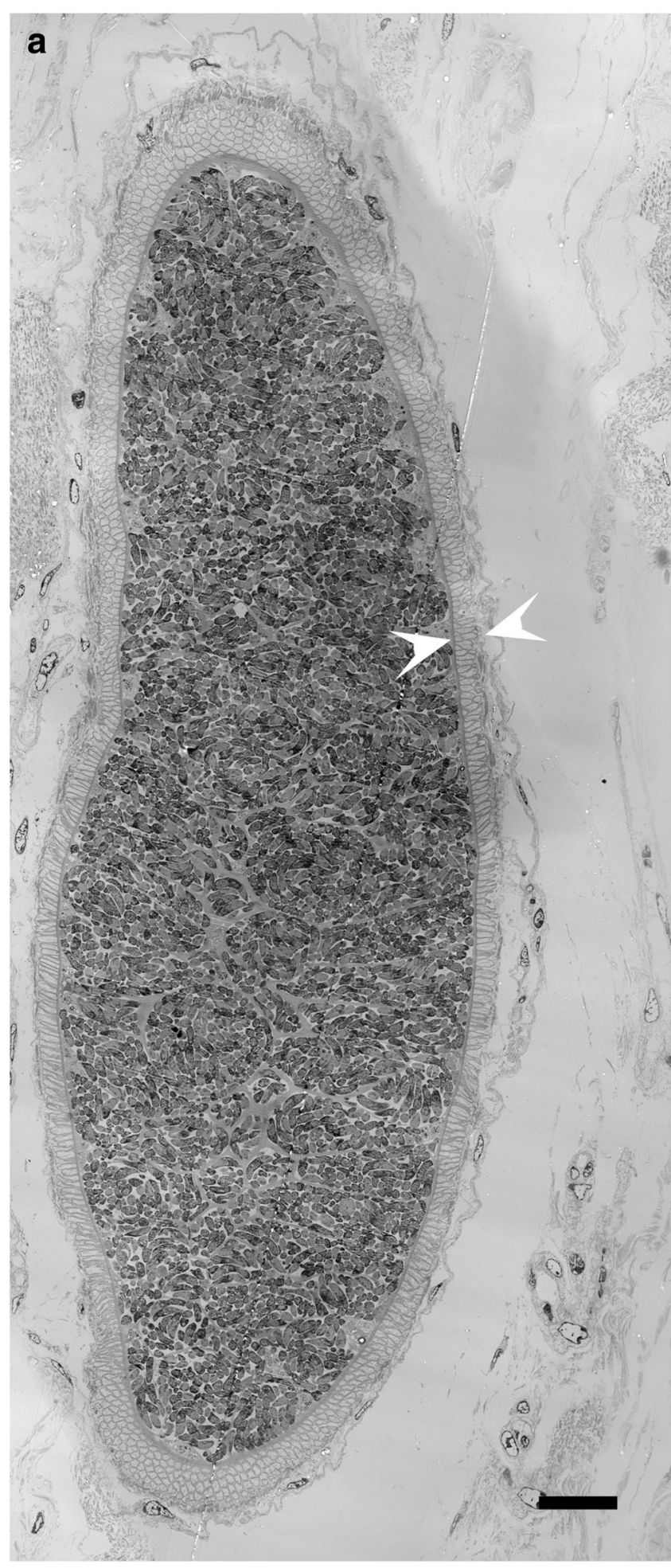

Fig. 8 TEM of subkind C Sarcocystis sp.2 sarcocysts. Scale bar in a $20 \mu \mathrm{m}$, in b, c $1 \mu \mathrm{m}$, d $100 \mathrm{~nm}$. Note thick sarcocyst wall, upright villar protrusions (vp), bradyzoites (br), metrocytes (me), ground substance (gs), microfilaments (mf), parasitophorous vacuolar membrane (pvm), electron-dense layer (edl), and plaque-like structures
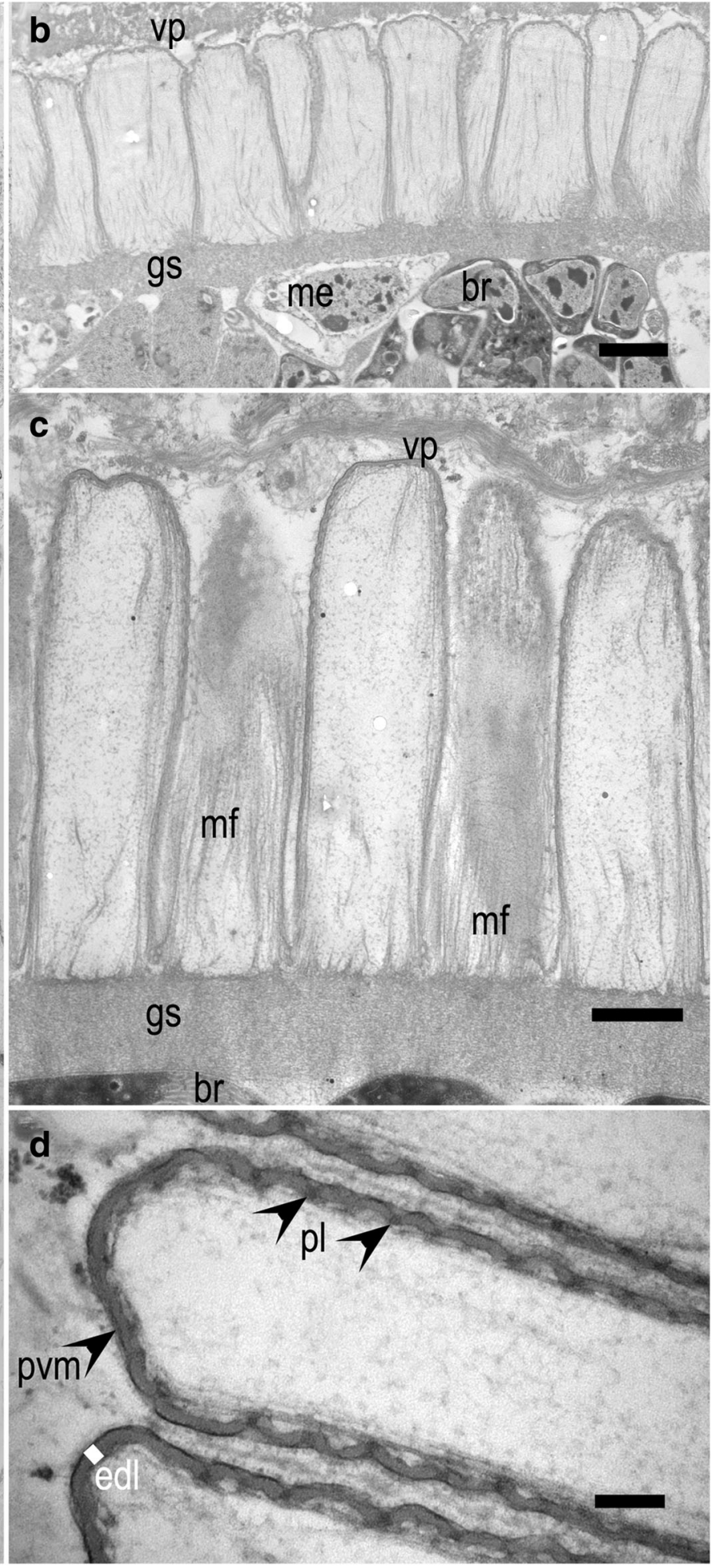

(pl). a Longitudinal section of a sarcocyst with thick wall (opposing arrowheads). b, c Note elongated vp with fine $\mathrm{mf}$ that are denser at the base. d Higher magnification of vp end to show wavy pvm, lined by edl, and $\mathrm{pl}$ 


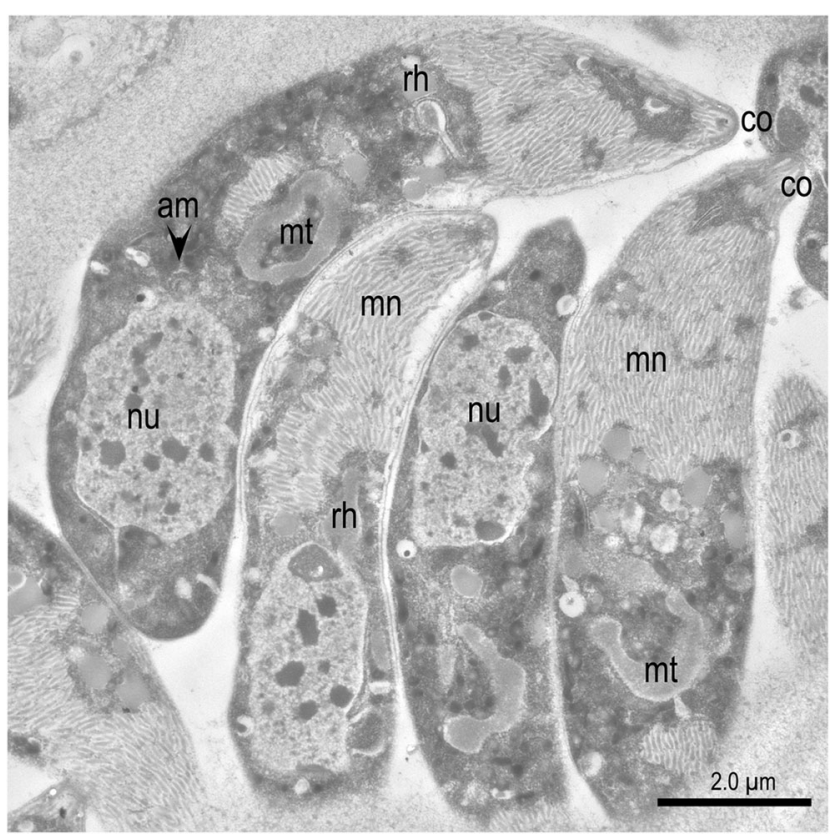

Fig. 9 TEM of subkind C Sarcocystis sp. 2 bradyzoites. Note conoid (co), numerous micronemes $(\mathrm{mn})$, a convoluted mitochondria $(\mathrm{mt})$, subterminal nucleus (nu), amylopectin granules (am), and rhoptries (rh)

\section{5 large ribosomal subunit DNA}

Over 1400 nucleotides were sequenced at the $28 \mathrm{~S}$ rDNA locus from three thick-walled samples. These sequences were most similar to those from $S$. truncata. Two sequences were nearly identical to published $S$. truncata $28 S$ rDNA sequences (99.9\% identity), while the third differed from $S$. truncata by 16 transitions, 2 transversions, and 4 indels encompassing 14 nucleotides total (97.4\% identity). No $28 S$ rDNA sequence from S. elongata was found in GenBank for comparison.

\section{ITS-1 sequencing}

The ITS- 1 locus was successfully sequenced for one thickwalled sarcocyst. Across $587 \mathrm{bp}$, the ITS-1 sequence was most similar to $S$. tarandi ( $99.1 \%$ identity). The next closest congeners were $S$. elongata, S. rangiferi, and S. truncata, sharing $95.1,86.6$, and $85.6 \%$ identity, respectively.

\section{Discussion}

This study revealed that Sarcocystis infection is common in the eastern elk in Pennsylvania. Sarcocysts were previously reported from two of two elk from Elk Island National Park, Alberta, Canada (Mahrt and Colwell 1980), and the Pacific northwest. Sayama (1952) found few sarcocysts in muscle from 19/36 (55\%) elk from California hunted in 1949. Pond and Speer (1979) found sarcocysts in 12/24 (50\%) elk from National Bison Range, Montana. In a histologic study, sarcocysts were detected in 178/184 (97\%) elk hunted in 1991-1992 from Oregon, including142/149 (95\%) hearts and 115/124 (93\%) tongues (Lagerquist and Foreyt 1993).

All of the elk sampled in our study appeared to be in good health and the histological examination revealed only mild myositis. Here, $97.4 \%$ of adults were infected, but it is noteworthy that eight of nine yearlings were also infected. Although only five calves were tested, none were infected, suggesting postnatal infection.

Among the Sarcocystis species in cervids, only Sarcocystis hemionilatrantis of mule deer (Odocoileus hemionis) is considered pathogenic (Dubey et al. 2016). Experimentally, mule deer fawns fed $S$. hemionilatrantis 50,000 or more sporocysts became ill and some died of acute sarcocystosis (Koller et al. 1977). Epidemiologic data indicates infection with $S$. hemionilatrantis affects growth of mule deer fawns (Dubey and Kistner 1985). Currently, there is no definitive evidence that any of the Sarcocystis species from wild cervids are transmissible to domestic livestock or humans (Dubey et al. 2016).

There is considerable confusion concerning the taxonomy of Sarcocystis species in cervids, based solely on molecular characteristics, and some Sarcocystis species were considered non-specific (reviewed in Dubey et al. 2016). In this study, DNA sequences from ribosomal loci clearly separated thickwalled sarcocysts from thin-walled, but the molecular differences between the samples reported here and other cervid Sarcocystis species were small, indicating that further sequencing is needed from multiple loci in order to accurately diagnose species based on DNA sequence. Furthermore, the North American elk is considered a separate species from red deer in Europe, and there are no transmission experiments to validate the specificity of Sarcocystis species among cervids. Therefore, we have not attempted to compare sarcocysts in elk with those of other cervids from America or Europe.

The ultrastructure of the sarcocyst wall is a useful taxonomic criterion for differentiating Sarcocystis species within a given host. Dubey et al. (2016) proposed 42 types of sarcocyst walls with many subdivisions within some of the wall types. It needs to be emphasized that this criterion may not be useful for comparing sarcocyst structure among Sarcocystis species of different hosts (Dubey et al. 2016). We are aware that in the present study, the TEM was performed on sarcocysts from paraffin blocks and thus the preservation was not ideal. However, the structure of the sarcocyst wall was fairly well preserved for differentiating Sarcocystis species.

Among four morphological types of sarcocysts found in this study, the most common was the thin-walled S. wapiti present in all infected elk. Based on TEM, it is distinct from other species in elk. It most closely resembles $S$. cervicanis (Hernández-Rodríguez et al. 1981a, b) from the red deer (Gjerde et al. 2017). However, the villar tips are bifurcated in $S$. cervicanis (Hernández-Rodríguez et al. 1981a, b) 


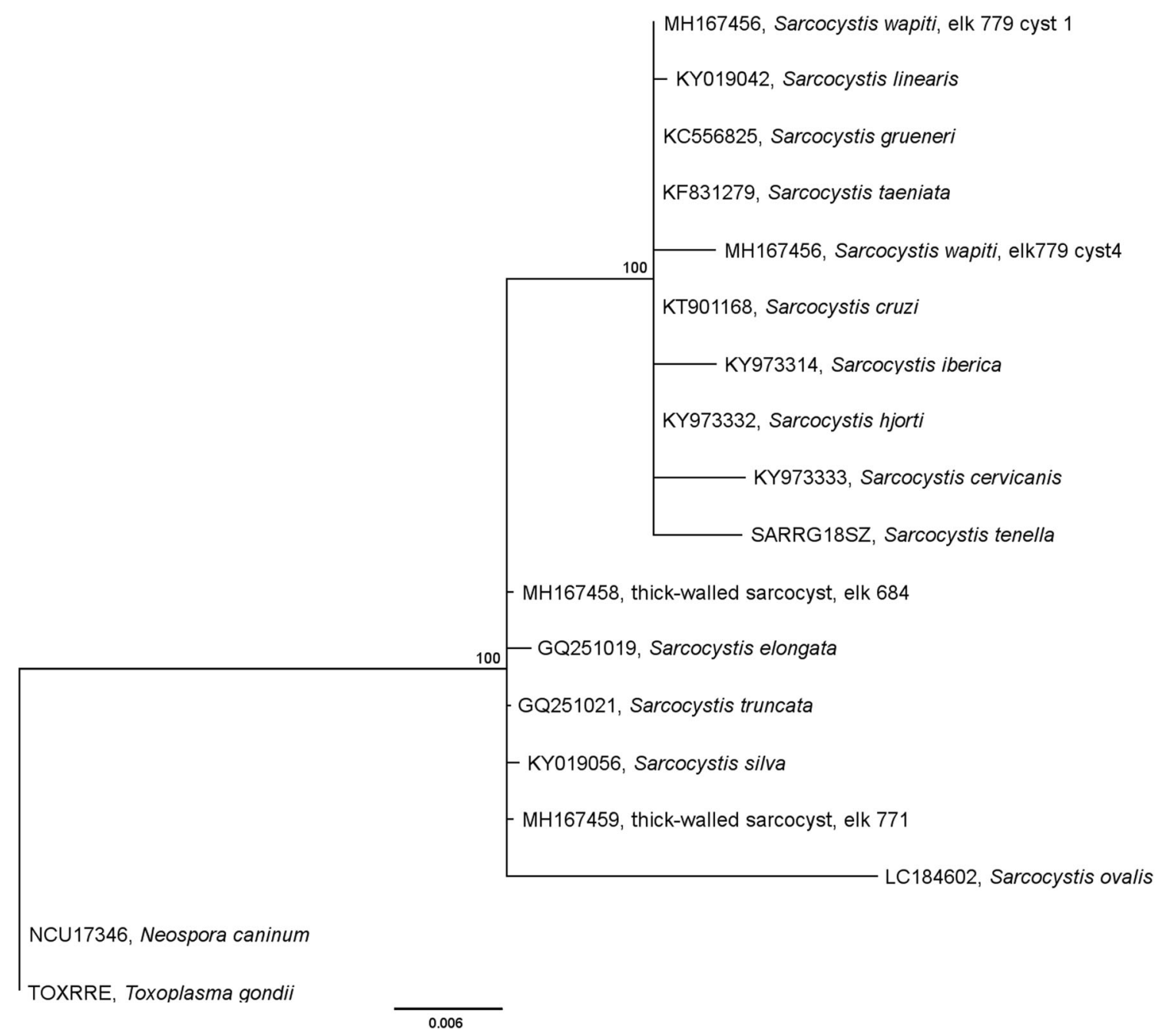

base of the clade with $S$. truncata, S. elongata, and S. silva, while the thinwalled $S$. wapiti is placed within a strongly supported sub-clade thick-walled sarcocysts were placed within a well-supported clade containing other cervid parasites. The thick-walled sarcocysts cluster at the

whereas they are not in $S$. wapiti. This observation was confirmed in the present study by TEM examination of seven sarcocysts. DNA sequence from the $18 \mathrm{~S} r D N A$ locus indicated that $S$. wapiti is closely related to $S$. taeniata and $S$. linearis, species found in European sika deer and roe deer, respectively, and all three are clearly distinct from S. cervicanis, differing at nearly $10 \%$ of nucleotides examined.

The presence of a second species of Sarcocystis, $S$. sybillensis-like described from elk in Wyoming and Oregon (Dubey et al. 1983; Lagerquist and Foreyt 1993), was confirmed in the present study in elk from Pennsylvania by the presence of thick-walled sarcocysts described as subkind A above. By light microscopy, the sarcocysts in the present study appeared similar to $S$. sybillensis from elk in Wyoming (Dubey et al. 1983). However, we did not see the lobulation/ branching at the base of vp depicted in Fig. 9 of Dubey et al. (1983). Further studies are needed to fully describe the structure of this species in optimally preserved specimens.
The third species, morphological subkind B Sarcocystis sp.1, was found only in one elk, and, thus, has not been given a species designation. Whether it is an accidental infection or a true parasite of elk needs further investigation.

The fourth species found, subkind C Sarcocystis sp.2, was the most commonly thick-walled Sarcocystis found in the present study. This species was clearly distinguished morphologically from the other thick-walled sarcocysts based on TEM; the villar protrusions on its wall were upright and long. The only mention of thick-walled sarcocysts from the elk outside of America is a mention of it in captive elk in a zoo in Germany by Stolte et al. (1996). They mentioned finding a Sarcocystis sp. with thick wall that had ear-like vp; TEM was not performed.

DNA sequencing indicated that all thick-walled sarcocysts in Pennsylvania elk are most closely related to $S$. truncata, $S$. elongata, and $S$. tarandi. It is worth noting that the highest similarity at all loci is found with $S$. truncata, a parasite originally described in European red deer (Gjerde et al. 2017). 
This suggests a common origin of these Sarcocystis lineages, given the relatively recent split of Eurasian and North American populations of C. elaphus (Polziehn and Strobeck 1998). The sarcocysts were not sorted to species prior to sequencing, so it is impossible to connect DNA sequences to specific species. Nevertheless, there appear to be two distinct sets of sequences within the three sarcocysts sequenced here. Sequences from two sarcocysts were nearly identical, while the third was consistently $\sim 3 \%$ diverged from the other two. This could be indicative of considerable intraspecific variation or the sequencing of two distinct species. Further sampling is required to distinguish among the alternatives. Regardless, all thickwalled species are within a strongly supported clade exclusive to cervid parasites.

Acknowledgements This research was supported in part by an appointment to the Agricultural Research Service (ARS) Research Participation Program administered by the Oak Ridge Institute for Science and Education (ORISE) through an interagency agreement between the US Department of Energy (DOE) and the US Department of Agriculture (USDA). ORISE is managed by ORAU under DOE contract number DE-SC0014664. All opinions expressed in this paper are the author's and do not necessarily reflect the policies and views of USDA, ARS, DOE, or ORAU/ORISE.

\section{Compliance with ethical standards}

Conflict of interest The authors declare that they have no conflict of interest.

Ethical statement The samples were obtained from legally hunted elk. No laboratory experiments were performed.

\section{References}

Cerqueira-Cézar CK, Thompson PC, Verma SK, Mowery J, CaleroBernal R, Murata FHA, Sinnett DR, van Hemert C, Rosenthal BM, Dubey JP (2017) Morphological and molecular characterization of Sarcocystis arctica-like sarcocysts from the Arctic fox (Vulpes lagopus) from Alaska, USA. Parasitol Res 116:18711878. https://doi.org/10.1007/s00436-017-5462-6

Dubey JP, Kistner TP (1985) Epizootiology of Sarcocystis infections in mule deer fawns in Oregon. J Am Vet Med Assoc 187:1181-1186

Dubey JP, Jolley WR, Thorne ET (1983) Sarcocystis sybillensis sp. nov. from the north American elk (Cervus elaphus). Can J Zool 61:737742. https://doi.org/10.1139/zB3-098

Dubey JP, van Wilpe E, Calero-Bernal R, Verma SK, Fayer R (2015) Sarcocystis heydorni n. sp. (Apicomplexa: Sarcocystidae) with cattle (Bos taurus) and human (Homo sapiens) cycle. Parasitol Res 114:4143-4147. https://doi.org/10.1007/s00436-015-4645-2

Dubey JP, Calero-Bernal R, Rosenthal BM, Speer CA, Fayer R (2016) Sarcocystosis of animals and humans, 2nd edn. CRC Press, Boca Raton, pp 1-481
Dubey JP, Brown J, Verma SK, Cerqueira-Cézar CK, Benefield J, Kwok OCH, Ying Y, Murata FHA, Pradhan AK, Su C (2017) Isolation of viable Toxoplasma gondii, molecular characterization, and seroprevalence in elk (Cervus canadensis) in Pennsylvania, USA. Vet Parasitol 243:1-5. https://doi.org/10.1016/j.vetpar.2017.05.030

Eveland J, George J, Hunter N, Forney D, Harrison R (1979) A preliminary evaluation of the ecology of the elk in Pennsylvania. In: Boyce M, Hayden-Wing L (eds) North American elk: ecology, behavior, and management. The University of Wyoming, Laramie, pp 145-151

Foreyt WJ, Baldwin TJ, Lagerquist JE (1995) Experimental infections of Sarcocystis spp. in rocky mountain elk (Cervus elaphus) calves. J Wildl Dis 31:462-466. https://doi.org/10.7589/0090-3558-31.4.462

Gjerde B, Schulze J (2014) Muscular sarcocystosis in two arctic foxes (Vulpes lagopus) due to Sarcocystis arctica n. sp.: sarcocyst morphology, molecular characteristics and phylogeny. Parasitol Res 113:811-821. https://doi.org/10.1007/s00436-013-3711-x

Gjerde B, Luzon M, Alunda JM, de la Fuente C (2017) Morphological and molecular characteristics of six Sarcocystis spp. from red deer (Cervus elaphus) in Spain, including Sarcocystis cervicanis and three new species. Parasitol Res 116:2795-2811. https://doi.org/ 10.1007/s00436-017-5590-z

Hernández-Rodríguez S, Martínez-Gómez F, Navarrete I, Acosta-García I (1981a) Estudio al microscopio optico y electronico del quiste de Sarcocystis cervicanis. Rev Ibérica Parasitol 41:351-361

Hernández-Rodríguez S, Navarrete I, Martínez-Gómez F (1981b) Sarcocystis cervicanis, nueva especie parásita del ciervo (Cervus elaphus). Rev Ibérica Parasitol 41:43-51

Koller LD, Kistner TP, Hudkins GG (1977) Histopathologic study of experimental Sarcocystis hemionilatrantis infection in fawns. Am J Vet Res 38:1205-1209

Lagerquist JE, Foreyt WJ (1993) Prevalence of Sarcocystis in elk (Cervus elaphus) in Oregon. Northwest Sci 67:196-198

Mahrt JL, Colwell DD (1980) Sarcocystis in wild ungulates in Alberta. J Wildl Dis 16:571-576

Polziehn RO, Strobeck C (1998) Phylogeny of wapiti, red deer, sika deer, and other north American Cervids as determined from mitocondrial DNA. Mol Phylogenet Evol 10:249-258

Pond DB, Speer CA (1979) Sarcocystis in free-ranging herbivores on the national bison range. J Wildl Dis 15:51-53

Sayama K (1952) Sarcocystis in deer and elk of California. Fish Game 38: 99-104

Speer CA, Dubey JP (1982) Sarcocystis wapiti sp. nov. from the American wapiti (Cervus elaphus). Can J Zool 60:881-888. https://doi.org/10.1139/z82-120

Stolte M, Bockhardt I, Odening K (1996) A comparative scanning electron microscopic study of the cyst wall in 11 Sarcocystis species of mammals. J Zool (Lond) 239:821-832. https://doi.org/10.1111/j. 1469-7998.1996.tb05480.x

Trupkiewicz JG, Calero-Bernal R, Verma SK, Mowery J, Davison S, Habecker P, Georoff TA, Ialeggio DM, Dubey JP (2016) Acute, fatal Sarcocystis calchasi-associated hepatitis in roller pigeons (Columbia livia f. dom.) at Philadelphia zoo. Vet Parasitol 216:5258. https://doi.org/10.1016/j.vetpar.2015.11.008

Williams SL, McLaren SB, Burgwin MA (1985) Paleo-archaeological and historical records of elected Pennsylvania mammals. Ann Carnegie Museum 54:77-188 